\title{
Low dose of Dasatinib in chronic myeloid leukemia
}

\begin{abstract}
The oncoprotein BCR-ABL1 helps with the diagnosis and monitoring the therapeutic response in patients with chronic myeloid leukemia. We describe the case of a 70-year-old woman with chronic myeloid leukemia who did not achieve a molecular response after 12 months of treatment. She was then managed with Dasatinib $70 \mathrm{mg}$ orally twice a day until she reached a complete molecular response. She developed pulmonary toxicity probably due to Dasatinib. The dosage was decreased to $70 \mathrm{mg}$ orally once a day. The patient continues with a complete molecular response with less side effects.

In chronic myeloid leukemia, as far as we know, there are no previous reports, after a molecular response with Dasatinib $70 \mathrm{mg}$ twice a day, the maintenance of a good molecular control with a single daily dose. This dose may allow a reduction in toxicity with a better quality of life for patients with chronic myeloid leukemia.
\end{abstract}

Volume 9 Issue 2 - 202 I

\author{
Jacobo Lester MD,' Enrique Klériga MD, ${ }^{2}$ \\ Fernando Pérez-Zincer $\mathrm{MD}^{3}$ \\ 'Department of Neurology, Instituto Mexicano de \\ Neurociencias, Hospital Ángeles Lomas. Huixquilucan, Estado de \\ México, México \\ ${ }^{2}$ Department of Neurosurgery, Instituto Mexicano de \\ Neurociencias, Hospital Ángeles Lomas, Huixquilucan, Estado de \\ México, México \\ ${ }^{3}$ Department of Hematology, Hospital Ángeles Lomas. \\ Huixquilucan, Estado de México, México
}

Correspondence: Jacobo Lester, MD,Vialidad de la Barranca \#22, Consultorio 750, Col.Valle de las Palmas C.P. 52787, Huixquilucan, Estado de México, México, Tel 52-55-5246-9790, Email jacobolester@hotmail.com

\section{Introduction}

The fusion of the Abelson murine leukemia gene (ABL1) on chromosome 9 with the breakpoint cluster region (BCR) gene on chromosome 22, known as the Philadelphia chromosome, has been postulated as the etiology of the chronic myeloid leukemia (CML). This translocation results in the expression of the oncoprotein called BCR-ABL1, causing leukemogenesis with a cell cycle and aberrant cytosine production. ${ }^{1}$ The recognition and measurement of this oncoprotein helps with the diagnosis and monitoring -the therapeutic response in patients with this disease. ${ }^{2}$

\section{Case report}

This is the case of a 69-year-old woman with a history of Gold IV chronic obstructive pulmonary disease secondary to heavy chronic smoking (120 packs per year for 40 years, stopped in 2010), Sjögren's syndrome diagnosed in 2013, seizures due to levofloxacin in 2014 and, chronic myeloid leukemia (CML) diagnosed in February 2017 studying leukocytosis in “check-up". In March 2017, she started oral treatment with Imatinib $400 \mathrm{mg}$ once a day with side effects such as: excessive fatigue, nausea and frequent vomiting, without being able to achieve molecular response (BCR / ABL1) 12 months after starting treatment. This was replaced by Dasatinib $70 \mathrm{mg}$ orally twice a day in March 2018. She presented better tolerance and reached a complete molecular response in October 2018. On January 14, 2019, the patient was hospitalized for pneumonia, multiple foci, with poor initial response to antibiotics. She maintained complete molecular response (BCR-ABL1 of $0.0 \%$ ) during this time. In addition to pneumonia, pulmonary toxicity due to Dasatinib was suspected and the Dasatinib dose was decreased to $70 \mathrm{mg}$ orally once a day. This Dasatinib dosage has been continued for 28 months after the CML entered in remission. The patient continues with a complete molecular response (BCRABL1 of $0.0 \%$ ).

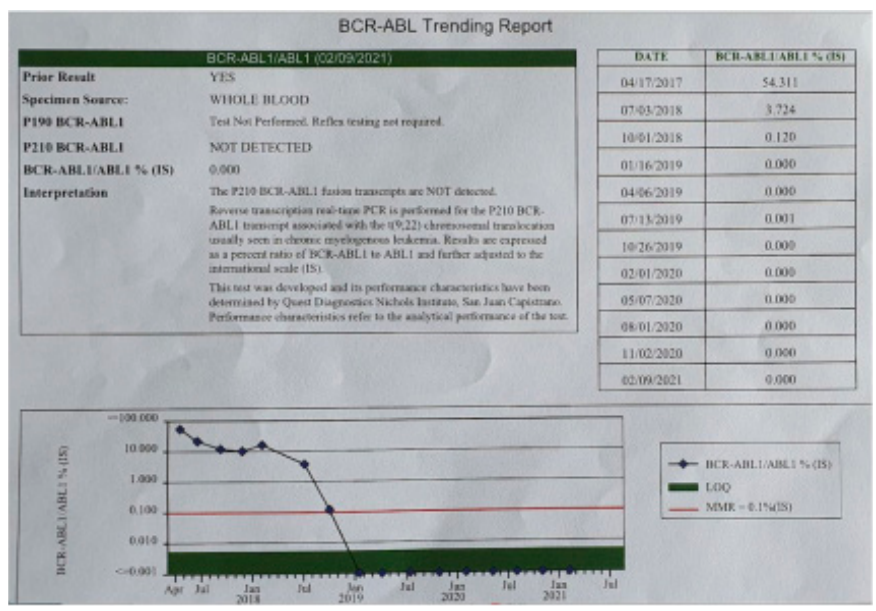

Figure I Report over time of BCR-ABLI.

Date of diagnosis: February 2017

Imatinib 400 mg once daily: March 2017 to March 2018

Dasatinib 70 mg twice a day: March 2018 to January 2019

Dasatinib $70 \mathrm{mg}$ once daily: January 2019 to present

Submission deadline: October 2018

Full remission: January 2019

Last measurement: February 202I.

\section{Discussion}

It is known that $50 \%$ of patients with CML are asymptomatic and are diagnosed by blood tests performed for some other reason, ${ }^{2}$ as has been the case in our patient. CML treatment - with tyrosine kinase inhibitors (TKI), interferes with the interaction of the BCR- 
ABL1 oncoprotein and adenosine triphosphate (ATP), blocking the cell proliferation of the malignant clone. The 10-year survival of the disease is about $80-90 \% .{ }^{2}$ Imatinib was the first TKI approved by the $\mathrm{FDA}^{3}$ and it is considered the treatment of choice with the usual dose of $400 \mathrm{mg}$ once a day with a 10 -year survival of $83.3 \%{ }^{4}$

Second-generation TKIs are more powerful but also have more adverse effects and toxicity than Imatinib. ${ }^{5}$ Dasatinib and Nilotinib are the second drugs generation indicated for failure or intolerance to Imatinib. Dasatinib dose is $70 \mathrm{mg}$ orally twice a day, ${ }^{2}$ however, there are reports that doses of $100 \mathrm{mg}$ once a day can induce a quick and profound response with a molecular response at 3 months. The response rate is $64 \%$ for Imatinib and $84 \%$ for Dasatinib, with a 5 -year overall survival practically the same, $91 \%$ and $90 \%$ respectively. ${ }^{6}$

The adverse effects of Dasatinib are apparently proportional to the dose, ${ }^{7}$ such as pleural effusion in $19 \%$, myelosuppression in $20 \%$ and pulmonary hypertension in $2 \%{ }^{8}$

A $70 \mathrm{mg}$ orally dose twice a day and $100 \mathrm{mg}$ once a day results in a similar response. ${ }^{8}$ When there is intolerance to Imatinib, Dasatinib should be considered. Comparative studies have been carried out with Dasatinib at different doses, $100 \mathrm{mg}$ once a day, $50 \mathrm{mg}$ twice a day, $140 \mathrm{mg}$ once a day and $70 \mathrm{mg}$ twice a day, with a 5-year total survival rate of $71-74-77-70 \%$ and progression-free survival of $49-51-40-47 \%$ with each dosage at 6 years of follow-up, respectively. ${ }^{9}$

Naqvi $\mathrm{K}$ et al. ${ }^{10}$ reported that Dasatinib, $50 \mathrm{mg}$ once a day in patients with newly diagnosed CML, 93\% achieved an early molecular response (MMR) at 3 months of follow-up with PCR for BCR-ABL1 $<10 \%$. Fluorescent in situ hybridization (FISH) showed that for $70 \%$ of patients the presence of this translocation was negative at 3 months; the remaining $30 \%$ did not make it negative for 6 months. ${ }^{10}$

Based on our experience, we propose the possibility of using Dasatinib $70 \mathrm{mg}$ twice a day until a complete molecular response is achieved and then reducing the dose to $70 \mathrm{mg}$ once a day to reduce the adverse effects of the drug.

\section{Conclusion}

To our knowledge, there are no previous reports that after achieving a complete molecular response in CML with Dasatinib, we may continue with a maintenance dose of $70 \mathrm{mg}$ orally every 24 hours. after reaching the complete molecular response with the standard dose of $70 \mathrm{mg}$ orally twice a day. We propose to explore Dasatinib $70 \mathrm{mg}$ orally as a long-term maintenance dose when there is toxicity at standard doses. This may allow a reduction in toxicity with a better quality of life for patients with CML. It is our believe that more studies should be done to confirm our findings.

\section{Conflicts of interest}

The authors declare that there are no conflicts of interest relevant to this work.

\section{Aknowledgments}

None.

\section{References}

1. Rowley JD. Letter: A new consistent chromosomal abnormality in chronic myelogenous leukaemia identified by quinacrine fluorescence and Giemsa staining. Nature. 1973;243(5405):290-293.

2. Jabbour E, Kantarjian H. Chronic myeloid leukemia: 2018 update on diagnosis, therapy and monitoring. Am J Hematol. 2018;93(3):442-459.

3. Druker BJ, Lydon NB. Lessons learned from the development of an abl tyrosine kinase inhibitor for chronic myelogenous leukemia. J Clin Invest. 2000;105(1):3-7.

4. Hochhaus A, Larson RA, Guilhot F, et al. Long-term outcomes of imatinib treatment for chronic myeloid leukemia. NEngl J Med. 2017;376(10):917927.

5. Radich JP, Kopecky KJ, Appelbaum FR, et al. A randomized trial of dasatinib $100 \mathrm{mg}$ versus imatinib $400 \mathrm{mg}$ in newly diagnosed chronicphase chronic myeloid leukemia. Blood. 2012;120(19):3898-3905.

6. Cortes JE, Saglio G, Kantarjian HM, et al. Final 5-year study results of DASISION: the dasatinib versus imatinib study in treatment naive chronic myeloid leukemia patients trial. J Clin Oncol. 2016;34(20):2333-2340.

7. Shanmuganathan N, Hiwase DK, Ross DM. Treatment of chronic myeloid leukemia: assessing risk, monitoring response, and optimizing outcome. Leuk Lymphoma. 2017;58(12):2799-2810.

8. Talpaz M, Shah NP, Kantarjian H, et al. Dasatinib in imatinib resistant Philadelphia chromosome-positive leukemias. $N$ Engl $J$ Med. 2006;354(24):2531-2541.

9. Shah NP, Guilhot F, Cortes JE, et al. Long-term outcome with dasatinib after imatinib failure in chronic-phase chronic myeloid leukemia: followup of a phase 3 study. Blood. 2014;123(15):2317-2324.

10. Naqvi K, Cortes JE, Skinner J, et al. Evaluating the Role of Lower Dose Dasatinib in Newly Diagnosed Early Chronic Phase-Chronic Myeloid Leukemia (CML-CP). Blood. 2017;130(suppl. 1):1611. 